Gut and Liver, Vol. 10, No. 5, September 2016, pp. 714-718

\title{
Clinicopathological Characteristics of Patients with Gastric Cancer according to the Expression of LIN28A
}

\author{
Chan Hyuk Park ${ }^{1}$, Jung Hwa Lee ${ }^{2}$, Na Keum Lee ${ }^{2}$, Yong Chan Lee ${ }^{2}$, and Sang Kil Lee ${ }^{2}$ \\ ${ }^{1}$ Department of Internal Medicine, Hanyang University Guri Hospital, Hanyang University College of Medicine, Guri, and ${ }^{2}$ Division of \\ Gastroenterology, Department of Internal Medicine, Severance Hospital, Institute of Gastroenterology, Yonsei University College of Medicine, \\ Seoul, Korea
}

Background/Aims: Although LIN28A is known to potentially play a role in the oncogenesis of various cancers, whether LIN28A expression is a predictor of poor prognosis in patients with gastric cancer has not been fully explored. We sought to evaluate clinicopathological characteristics according to the expression of LIN28A in numerous gastric cancer tissue samples. Methods: LIN28A expression was evaluated by immunohistochemical (IHC) analysis of a tissue microarray comprising 288 gastric cancer tissues and 288 adjacent normal tissues. Clinicopathological characteristics, including overall survival, were compared according to LIN28A expression. Results: The IHC staining score was lower for the cancer tissues than the normal tissues ( $p<0.001$ ). However, no significant differences were observed in the clinicopathological characteristics between the low and high LIN28A expression groups. In addition, the 5-year overall survival rate did not differ between the two groups: $75.3 \%$ (95\% confidence interval [Cl], 69.3\% to $81.7 \%$ ) versus $71.6 \%(95 \% \mathrm{Cl}, 63.3 \%$ to $80.9 \%$ ) for low versus high expression, respectively. Conclusions: The expression of LIN28A did not appear to play a distinct role in predicting the clinicopathological characteristics of patients with gastric cancer. In addition, LIN28A expression was not an independently associated factor for overall survival in patients with gastric cancer. (Gut Liver 2016;10:714-718)

Key Words: LIN28A; Stomach neoplasms; Survival

\section{INTRODUCTION}

While the survival rates of patients with early gastric cancer have improved with the increase of earlier diagnoses in Korea and Japan due to the use of endoscopy screening, ${ }^{1-4}$ the longterm oncologic outcomes of treatment in patients with advanced gastric cancer are still relatively poor. ${ }^{5,6}$ Although ongoing collaborative sequencing efforts have highlighted recurrent somatic genomic aberrations in gastric cancer, the outcomes of patients have not sufficiently improved. ${ }^{7}$ Better understanding of the molecular pathogenesis of gastric carcinogenesis may be needed for the advancement of gastric cancer treatments.

LIN28A is a highly conserved RNA-binding protein that was originally recognized as a key regulator of developmental timing in Caenorhabditis elegans. ${ }^{8}$ Recently, a study on LIN28A showed that it is overexpressed in various human cancers and functions as an oncogenes. ${ }^{9}$ p53 activates the expression of $m i R-34 a$ and $m i R-145$, which inhibit the expression of several stem-cell factors including OCT4, KLF4, LIN28A, and SOX2. ${ }^{10}$ Additionally, it has been shown that tristetraprolin induced by p53 in cancer cells increases the level of let-7, a known tumor suppressor, via downregulation of LIN28A. ${ }^{11}$ Blockade of the processing of various microRNAs, including let-7, has been suggested as one of the pathogenetic mechanisms of LIN28A in human carcinogenesis. ${ }^{12-15}$ In addition, LIN28A has been reported to suppress many other factors including OCT4, SOX2, and NANOG, which are involved in reprogramming human somatic cells, by post-transcriptional regulation. ${ }^{16,17}$

Expression of LIN28A has been identified in various human cancers, including breast cancer, ${ }^{18}$ ovarian cancer, ${ }^{19}$ hepatocellular carcinoma, ${ }^{9}$ and colorectal cancer. ${ }^{20}$ As for gastric cancer, one study from China showed that positive expression of LIN28A was correlated with poor overall survival. ${ }^{21}$ However, expression of LIN28A was found to be more common in corresponding normal tissue than in gastric cancer tissue in that study. In addition, the study did not fully adjust for TNM stages

Correspondence to: Sang Kil Lee

Division of Gastroenterology, Department of Internal Medicine, Yonsei University College of Medicine, 50-1 Yonsei-ro, Seodaemun-gu, Seoul 03722, Korea

Tel: +82-2-2228-1996, Fax: +82-2-393-6884, E-mail: sklee@yuhs.ac

Received on June 26, 2015. Revised on September 5, 2015. Accepted on September 8, 2015. Published online February 22, 2016 pISSN 1976-2283 eISSN 2005-1212 http://dx.doi.org/10.5009/gnl15283

@) This is an Open Access article distributed under the terms of the Creative Commons Attribution Non-Commercial License (http://creativecommons.org/licenses/by-nc/4.0) which permits unrestricted non-commercial use, distribution, and reproduction in any medium, provided the original work is properly cited. 
in the survival analysis. Therefore, we cannot be certain whether LIN28A expression is a poor prognostic factor in patients with gastric cancer. In the current study, we aimed to evaluate clinicopathological characteristics according to the expression of LIN28A by using a large number of gastric cancer tissue samples.

\section{MATERIALS AND METHODS}

\section{Study design and patient population}

In order to evaluate the relationship between the clinicopathological characteristics of gastric cancers and the expression of LIN28A, we performed immunohistochemical (IHC) staining for LIN28A using tissue microarrays (TMAs). The TMAs were constructed from 288 pairs of gastric cancer tissue samples and corresponding normal tissues that were obtained from patients who had undergone curative surgery for gastric cancer at the same center between April 2001 and December 2003. We collected the following data from the patients' medical records: demographic information, tumor location, histology, TNM staging, and overall survival duration. This study was approved by the Institutional Review Board of Severance Hospital.

\section{Tissue microarray construction}

The TMAs were constructed as previously described. ${ }^{22}$ On hematoxylin and eosin-stained slides of tumors, a representative area was selected, and the corresponding spot was marked on the surface of the paraffin block. Using a biopsy needle, the selected area was punched out, and a 2-mm tissue core was placed into an $8 \times 6$ recipient block. Gastric cancer tissues and corresponding normal tissues were then extracted. Each tissue core was assigned a unique TMA location number that was linked to a database containing other clinicopathological data.

\section{Immunohistochemistry}

IHC analyses were performed on formalin-fixed, paraffinembedded tissues, using the TMA. The slides were deparaffinized in xylene and rehydrated through an ethanol gradient. Endogenous peroxidase was blocked with 3\% $\mathrm{H}_{2} \mathrm{O}_{2}$ for 10 minutes. The slides were immersed in $10 \mathrm{mM}$ citrate buffer $(\mathrm{pH}$ 6.0) and heated for 10 minutes for antigen retrieval. Nonspecific binding was blocked by preincubation with a protein blocking agent (Immunotech Laboratories Inc., Monrovia, CA, USA), and the slides were incubated at room temperature for 1 hour with antibody against LIN28A (rabbit polyclonal, 1:500; Abcam, Cambridge, MA, USA). The samples were washed three times with phosphate-buffered saline (PBS) and then incubated with secondary antibodies for 30 minutes. After an additional three washes in PBS, the slides were incubated with the streptavidinhorseradish peroxidase complex (Dako, Glostrup, Denmark) for 30 minutes. The slides were visualized with diaminobenzidine (Dako) and then counterstained with hematoxylin. The expres- sion of the antibodies was assessed semiquantitatively by estimating the percentage of tumor cells with positive cytoplasm staining among the entire population of tumor cells. All slides were examined and scored independently by two experienced investigators to avoid subjective biases. Each slide was examined in its entirety under a light microscope, and a proportion score was initially assigned, which represented the estimated proportion of positive tumor cells ( 0 , none; $1,0 \%$ to $10 \%$; $10 \%$ to $50 \%$; and 3, 50\% to $100 \%$ ). Next, an intensity score was assigned, which represented the average intensity of the positive tumor cells (0, none; 1 , weak; 2 , intermediate; and 3, strong). The proportion and intensity scores were then multiplied to obtain a total score, which ranged from 0 to 9 , and high expression of LIN28A was defined as a total score $\geq 2 .^{21}$

\section{Statistical analysis}

Statistical tests used to compare the measured results included the t-test, Mann-Whitney U test, chi-square test, and Fisher exact test. The Kaplan-Meier method and log-rank test were used for survival analysis. In addition, the Cox proportional hazards model was used to adjust for possible confounding variables including TNM stage. A value of $p$ less than 0.05 was regarded as a statistically significant difference for comparisons between groups. All statistical procedures were conducted using the statistical software SPSS for Windows version 18.0 (SPSS Inc., Chicago, IL, USA) with the exception of the survival analysis, which was performed using R version 2.15.3 (R Foundation for Statistical Computing, Vienna, Austria).

\section{RESULTS}

\section{Comparison of LIN28A expression between cancer tissues and corresponding normal tissues}

IHC staining was performed on 288 pairs of normal and cancer tissue samples to analyze the difference in LIN28A expression. The mean IHC staining score was higher in the normal tissues than in the cancer tissues (normal vs cancer [mean \pm standard deviation], $2.0 \pm 1.6$ vs $1.1 \pm 1.5 ; \mathrm{p}<0.001)$. The proportion of tissues with LIN28A expression was higher in the normal tissues than in the cancer tissues (normal vs cancer, 63.5\% vs 35.4\%; $\mathrm{p}<0.001$ ).

\section{LIN28A expression and clinicopathological characteristics of gastric cancer}

The clinicopathological characteristics of the enrolled patients according to the LIN28A expression of cancer tissues on IHC staining are shown in Table 1. High expression of LIN28A was shown in 102 of 288 patients (35.4\%). The mean patient age and the proportion of men did not differ between the groups ( $p=0.469$ and $p=0.217$, respectively). Neither tumor location nor histology differed between the groups $(\mathrm{p}=0.148$ and $\mathrm{p}=0.827$, respectively). In addition, T, N, and TNM stages did not differ 
Table 1. Clinicopathological Characteristics according to LIN28A Expression via Immunohistochemical Staining

\begin{tabular}{|c|c|c|c|}
\hline Variable & $\begin{array}{l}\text { Low expression on IHC staining } \\
\text { (IHC staining score }<2)(n=186)\end{array}$ & $\begin{array}{l}\text { High expression on IHC staining } \\
\text { (IHC staining score } \geq 2)(n=102)\end{array}$ & p-value \\
\hline Age, yr & $56.2 \pm 12.1$ & $55.1 \pm 12.8$ & 0.469 \\
\hline Sex & & & 0.217 \\
\hline Male & $116(62.4)$ & 56 (54.9) & \\
\hline Female & 70 (37.6) & $46(45.1)$ & \\
\hline Location & & & 0.148 \\
\hline Upper third & 34 (18.3) & $29(28.4)$ & \\
\hline Middle third & 34 (18.3) & 14 (13.7) & \\
\hline Lower third & 115 (61.8) & 59 (57.8) & \\
\hline Anastomosis site & $3(1.6)$ & 0 & \\
\hline Histology & & & 0.827 \\
\hline AWD or AMD & 45 (24.2) & $28(27.5)$ & \\
\hline APD or SRC & $129(69.4)$ & $68(66.7)$ & \\
\hline Others* & $12(6.5)$ & $6(5.9)$ & \\
\hline T stage & & & 0.284 \\
\hline pT1 & $3(1.6)$ & 0 & \\
\hline pT2 & 31 (16.7) & $25(24.5)$ & \\
\hline pT3 & 65 (34.9) & 34 (33.3) & \\
\hline pT4 & $87(46.8)$ & $43(42.2)$ & \\
\hline $\mathrm{N}$ stage & & & 0.375 \\
\hline pNo & 74 (39.8) & 36 (35.3) & \\
\hline $\mathrm{pN} 1$ & 54 (29.0) & $25(24.5)$ & \\
\hline $\mathrm{pN} 2$ & 32 (17.2) & $26(25.5)$ & \\
\hline $\mathrm{pN} 3$ & $26(14.0)$ & 15 (14.7) & \\
\hline TNM stage & & & 0.725 \\
\hline IIa & 59 (31.7) & $30(29.4)$ & \\
\hline $\mathrm{IIb}$ & 65 (34.9) & 34 (33.3) & \\
\hline IIIIa & $28(15.1)$ & 19 (18.6) & \\
\hline $\mathrm{IIIb}$ & $14(7.5)$ & $11(10.8)$ & \\
\hline IIIC & 20 (10.8) & $8(7.8)$ & \\
\hline
\end{tabular}

Data are presented as mean \pm SD or number (\%).

IHC, immunohistochemical; AWD, adenocarcinoma well differentiated; AMD, adenocarcinoma moderated differentiated; APD, adenocarcinoma poorly differentiated; SRC, signet ring cell carcinoma.

*This category included mucinous adenocarcinoma, lymphoepithelioma-like carcinoma, and glandular endocrine carcinoma.

between the groups $(p=0.284, p=0.375$, and $p=0.725$, respectively).

\section{Survival analysis according to LIN28A expression}

Kaplan-Meier plots were used to illustrate overall survival according to the expression of LIN28A (Fig. 1). The median followup durations were 69.4 months (interquartile range [IQR], 60.6 to 77.4 months) and 66.9 months (IQR, 40.8 to 76.7 months) in the low and high expression groups, respectively ( $p=0.158$ ). The 5-year overall survival was 75.3\% (95\% confidence interval [CI], $69.3 \%$ to $81.7 \%$ ) in the low expression group and $71.6 \%$ (95\% CI, $63.3 \%$ to $80.9 \%$ ) in the high expression group. The duration of overall survival did not differ between the groups $(p=0.405)$.
A Cox proportional hazards model showed that high LIN28A expression was not an independently associated factor for death after adjusting for TNM stage (hazard ratio, 1.191; 95\% CI, 0.758 to 1.872 ) (Table 2).

\section{DISCUSSION}

LIN28A has been considered an oncogene in the development of various human cancers. ${ }^{9,18-20}$ One of the possible mechanisms of LIN28A as an oncogene is downregulation of the let-7 family, well-known microRNAs that function as tumor suppressors. ${ }^{12-14}$ Under this hypothesis, Xu et al ${ }^{21}$ performed a study on the expression of LIN28A in gastric cancer patients via real- 


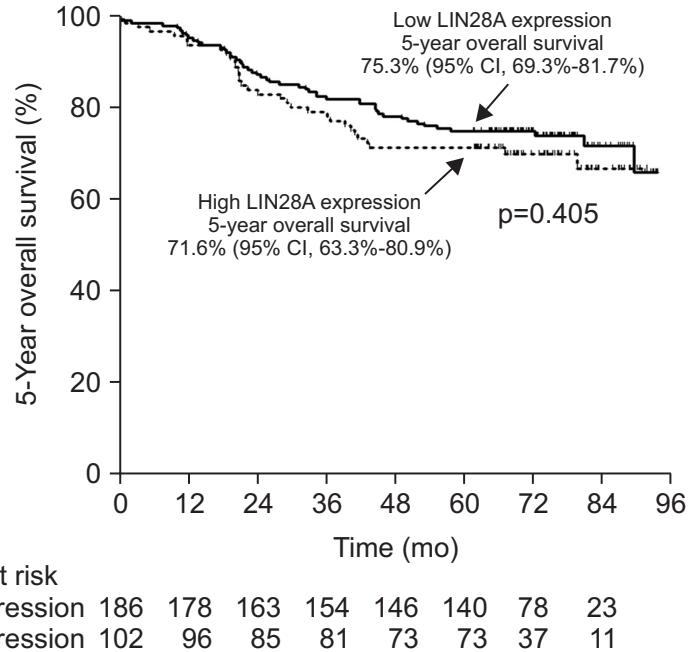

Fig. 1. Kaplan-Meier plots for the overall survival rate according to LIN28A expression. The median follow-up durations of the low- and high-expression groups were 69.4 months (interquartile range [IQR], 60.6 to 77.4 months) and 66.9 months (IQR, 40.8 to 76.7 months), respectively.

time polymerase chain reaction analysis and IHC staining. In their study, however, the expression level of LIN28A in normal tissues was higher than that in cancer tissues (normal vs cancer, $62.8 \%$ vs $46.0 \%$ ). Although it was shown that LIN28A expression was independently associated with poor overall survival, the authors could not clearly explain how LIN28A expression affected the overall survival of patients with gastric cancer. We questioned whether the expression of LIN28A really has a negative influence on overall survival in patients with gastric cancer.

In our study, similar proportions of cancer tissues and corresponding normal tissues showed expression of LIN28A on IHC staining (normal vs cancer, 63.5\% vs 35.4\%) compared to the results of Xu et al. ${ }^{21}$ However, no associated clinicopathological factor for the high LIN28A expression in gastric cancers was identified. Unlike Xu et al., ${ }^{21}$ we classified gastric cancers into four or five groups according to T stage (pT1, pT2, pT3, and pT4), N stage (pNo, pN1, pN2, and pN3), and TNM stage (IIa, Ilb, IIIIa, IIIlb, and IIIc). These detailed classifications were selected to stratify the gastric cancers more accurately for survival analysis. As a result, we showed that LIN28A expression was not an independently associated factor for overall survival. The expression of LIN28A seemed to have no distinct role in predicting clinicopathological characteristics including survival outcomes in patients with gastric cancer.

Our results, which are in conflict with the usual expectation that LIN28A may have a role as an oncogene, are likely due to the various roles of LIN28A aside from repressing let-7. First, overexpression of LIN28A induces the expression of several transcription factors that are involved in early embryonic-cell fate decisions. Moreover, LIN28A functions independently of let-7. ${ }^{23}$ Second, LIN28A directly regulates various metabolisms
Table 2. Cox Proportional Hazards Model for Predicting Death

\begin{tabular}{lcr}
\hline \multicolumn{1}{c}{ Variable } & Hazard ratio $(95 \% \mathrm{CI})$ & $\mathrm{p}$-value \\
\hline TNM stage & 1 & \\
IIa & $1.361(0.672-2.757)$ & 0.392 \\
IIb & $3.302(1.608-6.779)$ & 0.001 \\
IIIa & $5.172(2.340-11.434)$ & $<0.001$ \\
IIIb & $7.897(3.826-16.296)$ & $<0.001$ \\
IIIc & $1.191(0.758-1.872)$ & 0.448 \\
High LIN28A expression & & \\
\hline
\end{tabular}

CI, confidence interval.

including oxidative phosphorylation or glucose metabolism. ${ }^{24,25}$ Third, LIN28A may facilitate the global translational suppression of endoplasmic reticulum-associated mRNAs in undifferentiated stem cells. ${ }^{26}$ We believe that LIN28A may have a role not only in gastric cancer cells but also in normal gastric cells. In addition, the heterogeneity of gastric cancers could contribute to the discrepancy between the results of Xu et al.'s study and ours. $^{27,28}$ A better understanding of the molecular biology of gastric cancers and appropriate selection of the patient population might be necessary to clarify the role of LIN28A in gastric carcinogenesis.

Although this was the largest study to date that evaluated clinicopathological characteristics according to the expression of LIN28A in patients with gastric cancer, it has several limitations. First, we did not assess the lesion characteristics according to well-known associated factors for gastric cancer, including Helicobacter pylori infection status and family history of gastric cancer. Unfortunately, H. pylori infection status and family history were unavailable in our gastric cancer cohort for TMAs. Second, various associated factors for LIN28A including p53, Ki-67, miR-34a, miR-145, and let-7, were not analyzed in the study. Evaluating these factors might support the negative results of our study in patients with gastric cancer. The lack of an analysis of LIN28B was another limitation of the study. Although the study was performed to validate the results of the previous study, ${ }^{21}$ expression of LIN28B should be investigated to better understand LIN28 in patients with gastric cancer. ${ }^{29,30}$ Despite these limitations, our data may form the basis of a system that can be used to understand the clinicopathological characteristics based on LIN28A expression.

The expression of LIN28A seemed to have no distinct role in predicting clinicopathological characteristics in gastric cancer. Additionally, LIN28A expression was not an independently associated factor for overall survival in patients with gastric cancer.

\section{CONFLICTS OF INTEREST}

No potential conflict of interest relevant to this article was reported. 


\section{ACKNOWLEDGEMENTS}

This research was supported by the Basic Science Research Program through the National Research Foundation of Korea funded by the Ministry of Education, Science and Technology (2012R1A1A2001479).

\section{REFERENCES}

1. Lee HJ, Yang HK, Ahn YO. Gastric cancer in Korea. Gastric Cancer 2002;5:177-182

2. Isobe $\mathrm{Y}$, Nashimoto A, Akazawa $\mathrm{K}$, et al. Gastric cancer treatment in Japan: 2008 annual report of the JGCA nationwide registry. Gastric Cancer 2011;14:301-316.

3. Carter KJ, Schaffer HA, Ritchie WP Jr. Early gastric cancer. Ann Surg 1984;199:604-609.

4. Shimizu S, Tada M, Kawai K. Early gastric cancer: its surveillance and natural course. Endoscopy 1995;27:27-31.

5. Cunningham D, Starling N, Rao S, et al. Capecitabine and oxaliplatin for advanced esophagogastric cancer. N Engl J Med 2008;358:36-46.

6. Waddell T, Chau I, Cunningham D, et al. Epirubicin, oxaliplatin, and capecitabine with or without panitumumab for patients with previously untreated advanced oesophagogastric cancer (REAL3): a randomised, open-label phase 3 trial. Lancet Oncol 2013;14:481489.

7. Yang W, Raufi A, Klempner SJ. Targeted therapy for gastric cancer: molecular pathways and ongoing investigations. Biochim Biophys Acta 2014;1846:232-237.

8. Moss EG, Lee RC, Ambros V. The cold shock domain protein LIN28 controls developmental timing in C. elegans and is regulated by the lin-4 RNA. Cell 1997;88:637-646.

9. Viswanathan SR, Powers JT, Einhorn W, et al. Lin28 promotes transformation and is associated with advanced human malignancies. Nat Genet 2009;41:843-848.

10. Jain AK, Allton K, Iacovino M, et al. p53 regulates cell cycle and microRNAs to promote differentiation of human embryonic stem cells. PLoS Biol 2012;10:e1001268.

11. Lee JY, Kim HJ, Yoon NA, et al. Tumor suppressor p53 plays a key role in induction of both tristetraprolin and let-7 in human cancer cells. Nucleic Acids Res 2013;41:5614-5625.

12. Nam Y, Chen C, Gregory RI, Chou JJ, Sliz P. Molecular basis for interaction of let-7 microRNAs with Lin28. Cell 2011;147:10801091.

13. Leung AK, Sharp PA. MicroRNA functions in stress responses. 2010;40:205-215.

14. Newman MA, Thomson JM, Hammond SM. Lin-28 interaction with the Let-7 precursor loop mediates regulated microRNA processing. RNA 2008;14:1539-1549.

15. Mao XG, Hütt-Cabezas M, Orr BA, et al. LIN28A facilitates the transformation of human neural stem cells and promotes glioblastoma tumorigenesis through a pro-invasive genetic program. Oncotarget 2013;4:1050-1064.

16. Qiu C, Ma Y, Wang J, Peng S, Huang Y. Lin28-mediated posttranscriptional regulation of Oct4 expression in human embryonic stem cells. Nucleic Acids Res 2010;38:1240-1248.

17. Yu J, Vodyanik MA, Smuga-Otto K, et al. Induced pluripotent stem cell lines derived from human somatic cells. Science 2007; 318:1917-1920.

18. Lv K, Liu L, Wang L, et al. Lin28 mediates paclitaxel resistance by modulating $\mathrm{p} 21, \mathrm{Rb}$ and Let-7a miRNA in breast cancer cells. PLoS One 2012;7:e40008.

19. Peng S, Maihle NJ, Huang Y. Pluripotency factors Lin28 and Oct4 identify a sub-population of stem cell-like cells in ovarian cancer. Oncogene 2010;29:2153-2159.

20. Saiki Y, Ishimaru S, Mimori K, et al. Comprehensive analysis of the clinical significance of inducing pluripotent stemness-related gene expression in colorectal cancer cells. Ann Surg Oncol 2009; 16:2638-2644.

21. Xu C, Shen J, Xie S, Jiang Z, Huang L, Wang L. Positive expression of Lin28 is correlated with poor survival in gastric carcinoma. Med Oncol 2013;30:382.

22. Rimm DL, Camp RL, Charette LA, Costa J, Olsen DA, Reiss M. Tissue microarray: a new technology for amplification of tissue resources. Cancer J 2001;7:24-31.

23. Balzer E, Heine C, Jiang Q, Lee VM, Moss EG. LIN28 alters cell fate succession and acts independently of the let-7 microRNA during neurogliogenesis in vitro. Development 2010;137:891-900.

24. Zhu H, Shyh-Chang N, Segrè AV, et al. The Lin28/let-7 axis regulates glucose metabolism. Cell 2011;147:81-94.

25. Shyh-Chang N, Zhu H, Yvanka de Soysa T, et al. Lin28 enhances tissue repair by reprogramming cellular metabolism. Cell 2013; 155:778-792

26. Cho J, Chang H, Kwon SC, et al. LIN28A is a suppressor of ERassociated translation in embryonic stem cells. Cell 2012;151:765777

27. Das K, Gunasegaran B, Tan IB, Deng N, Lim KH, Tan P. Mutually exclusive FGFR2, HER2, and KRAS gene amplifications in gastric cancer revealed by multicolour FISH. Cancer Lett 2014;353:167175.

28. Nishida Y, Kuwata T, Nitta H, et al. A novel gene-protein assay for evaluating HER2 status in gastric cancer: simultaneous analyses of HER2 protein overexpression and gene amplification reveal intratumoral heterogeneity. Gastric Cancer 2015;18:458-466.

29. Wang T, Wang G, Hao D, et al. Aberrant regulation of the LIN28A/LIN28B and let-7 loop in human malignant tumors and its effects on the hallmarks of cancer. Mol Cancer 2015;14:125.

30. Piskounova E, Polytarchou C, Thornton JE, et al. Lin28A and Lin28B inhibit let-7 microRNA biogenesis by distinct mechanisms. Cell 2011;147:1066-1079. 